\title{
Pensamiento de los profesores de historia acerca de la ciudadanía y la inclusión de las movilizaciones estudiantiles en su enseñanza*
}

\author{
History teachers' thinking on citizenship and the inclusion of the student \\ mobilizations into their teaching \\ Pensamento dos professores de história a respeito da cidadania e \\ a inclusão das mobilizações estudiantiles em seu ensino \\ Cavieres Fernández, Eduardo ${ }^{a}$; Muñoz Reyes, José Antonio ${ }^{b}$

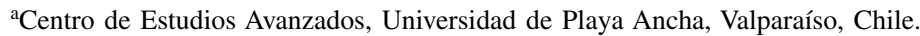 \\ Teléfono: 322624710 - 2205927. Correo Electrónico: ecavieres-cea@upla.cl

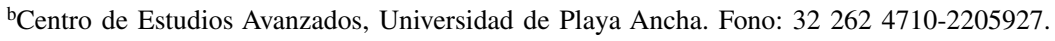 \\ Correo electrónico: jose.munoz-cea@upla.cl
}

\begin{abstract}
RESUMEN
En Chile los estudiantes secundarios participan activamente en movilizaciones estudiantiles. En esta investigación se indagó la posible relación entre el pensamiento de los profesores acerca de la ciudadanía y la inclusión de esta temática acerca de las movilizaciones estudiantiles en su enseñanza. Se aplicaron cuestionarios autoelaborados a 152 profesores de historia de enseñanza secundaria. Los resultados indican que los profesores incluyen mayoritariamente las movilizaciones estudiantiles en el currículo que enseñan. Ello se relaciona con el pensamiento de los profesores, ya que esta inclusión se asoció significativamente a la valoración positiva que dieron los profesores a estas movilizaciones, lo que a su vez se asoció de manera robusta con su adhesión teórica a una perspectiva colectiva respecto de la ciudadanía. El énfasis en la relevancia del pensamiento de los profesores del presente estudio resalta un recurso pedagógico que facilita la incorporación al aula de una temática afín a los estudiantes.
\end{abstract}

Palabras clave: pensamiento de los profesores, movilizaciones estudiantiles, ciudadanía, contenidos de enseñanza.

\begin{abstract}
Chilean secondary students participate actively in student mobilizations. This research study explored the relationship between teachers' opinions on citizenship and the inclusion of the topic of students' mobilizations into their teaching practice. A survey was given to 152 secondary history teachers throughout the country. Results indicate that (a) a majority of teachers included the issue of the students' mobilizations in their lectures; (b) there was a relationship between the teacher appreciation of the students' mobilizations and the inclusion of this matter in their teaching; and (c) this appreciation, in turn, is associated with the teachers' theoretical support to a collective vision of citizenship. The focus on the importance of teachers' perspectives, in this study, is considered and promoted as a valuable pedagogic resource for the inclusion of issues that are relevant to students.
\end{abstract}

Key words: teacher's thinking, student mobilizations, citizenship, teaching content.

\section{RESUMO}

No Chile, os estudantes da Educação Secundária Obrigatória participam ativamente das mobilizações estudantis. Questionou-se a possível relação entre o pensamento dos professores a respeito da cidadania e da inclusão de esta temática em seu ensino. Aplicaram-se questionários autoelaborados a 152 professores de História da Educação 
Secundária. Resultados indicam que os professores incluem principalmente as mobilizações estudantis nos contúdos que ensinam. Isso tem a ver com o pensamento dos professores já que esta inclusão se relacionou significativamente com valoração positiva que deram os professores a tais mobilizações, o que, por sua vez, associou-se fortemente com a adesão teórica a uma perspectiva coletiva de cidadania. A ênfase na relevância do pensamento dos professores no presente estudo ressalta um recurso pedagógico facilitador da incorporação de um temática de interesse dos estudantes na aula.

Palavras chave: pensamento dos professores, mobilizações estudantis, cidadania, conteúdos de ensino.

\section{INTRODUCCIÓN}

La literatura asigna gran importancia al rol de los profesores de facilitar recursos y estrategias para que los estudiantes se formen cívicamente (Torney-Purta \& Richardson, 2002; Pace, 2008; Ochoa, 2011). Entre otros aspectos, el pensamiento de los profesores respecto de temas de ciudadanía, construido sobre la base de sus propias concepciones acerca de qué significa ser un buen ciudadano y en relación con los contextos políticos y culturales en los que se desenvuelven, contribuye a enmarcar la formación ciudadana que entregan a sus alumnos (Davies, Gregory \& Riley, 1999; Bishop \& Hamot, 2001; Westheimer \& Kahne, 2004; Chin \& Barber, 2010). La relevancia de estos contextos para la formación ciudadana de los estudiantes ha sido igualmente subrayada por las investigaciones en el área (Shultz, Ainley, Frailon, Kerr \& Losito, 2010).

Actualmente en Chile, el contexto escolar ha estado profundamente marcado por movilizaciones estudiantiles, las que han impactado de manera especial a los estudiantes de educación secundaria (OPECH, 2010). Los estudiantes chilenos, junto a varias demandas educativas, han protestado tanto contra el rol del Estado como en contra del modelo de desarrollo que ha adoptado el país, y han promovido un ejercicio cívico activo (Cabalin, 2012; Urra, 2012). Desde esa perspectiva, las movilizaciones estudiantiles podrían estar ofreciendo un contexto de desarrollo ciudadano importante para los estudiantes. Varios de estos aspectos han sido tangencialmente incorporados a las directrices curriculares sugeridas por el Ministerio de Educación referidos a la formación ciudadana (MINEDUC, 2009). No obstante, no existen estudios que señalen si estos contenidos han sido efectivamente incorporados al currículo.

Debido a su cercanía con el área de la formación ciudadana, es de interés conocer si los profesores de Historia incluyen esta temática de las movilizaciones estudiantiles en su enseñanza y si en ello incide su propio pensamiento acerca de la ciudadanía. Por tanto, el objetivo de este estudio consiste en indagar la relación que existe entre el pensamiento de los profesores de historia acerca de la ciudadanía y la inclusión de esta temática de las movilizaciones estudiantiles en su enseñanza. Para considerar el pensamiento de los profesores se ha considerado su adhesión a alguna perspectiva teórica de ciudadanía, su adhesión al ejercicio cívico activo, y su valorización de las movilizaciones estudiantiles como contexto de desarrollo cívico.

\subsection{PERSPECTIVAS SOBRE LA CIUDADANÍA}

La noción de ciudadanía está estrechamente vinculada a los modos de participación que se establecen en determinadas unidades políticas soberanas y que, por tanto, determinan el modo cómo los sujetos se relacionan entre sí para decidir respecto de su autogobierno 
(Van Steenberger, 1994). Estos modos de participación han sido descritos en la literatura conformando distintas perspectivas en la ciudadanía que integran formas diversas de comprender la vida social, en general, y la vida democrática, en particular (Beiner, 1995; Habermas, 1996). En líneas generales, estas perspectivas enfatizan alguno de los dos polos de la vida social que suelen estar en tensión: el individuo y la colectividad (Walzer, 1990; Van Gunsteren, 1994). Esta tensión, a su vez, suele relacionarse con dos temas que son centrales para las naciones modernas: su desarrollo económico y el rol del Estado (Touraine, 1997).

Por un lado, propulsores de una perspectiva individualista (Held, 2006) enfatizan al individuo y sus derechos inalienables, los que no pueden ser coaptados por la colectividad. En su vertiente más influyente se propone la preeminencia del individuo por sobre la existencia de un bien común; a lo más, dicho bien común representa la suma agregada de los intereses individuales de los ciudadanos (Schumpeter, 1976). Este individualismo no se refiere necesariamente a individuos completamente autónomos, sino que supone la incorporación de estos a grupos o asociaciones instrumentales que avancen sus intereses privados o particulares (Bobbio, 1987). Esta perspectiva promueve la resolución de conflictos sociales mediante mecanismos electorales para que los ciudadanos puedan negociar competitivamente sus diferencias individuales, sin que se requiera la intervención unilateral del Estado (Becker, 1976). Junto a ello, los ciudadanos exigen al Estado resguardar su libertad para desarrollar sus intereses privados, los que deberían redundar en el desarrollo social (Hayek, 1960).

Por el otro lado, quienes promueven la perspectiva colectiva insisten en la centralidad de la comunidad y la cooperación (Gould, 1990). En sus líneas generales, este énfasis en la colectividad se opone a la desigualdad que se origina en las relaciones de competencia establecidas entre los individuos para superar sus diferencias (Mouffe, 2005). Esta perspectiva más bien aboga por un espacio público en donde los ciudadanos participan activamente para deliberar en torno a sus diferencias y acordar metas y prácticas que favorezcan el bien común (Young, 1996; Barber, 2003). De esta forma, el ámbito político tiene preeminencia por sobre el ámbito privado-económico, pues permite garantizar la participación de los ciudadanos (Macpherson, 1977). Por ello, los ciudadanos deben exigir al Estado promover el bien común, cuyo desarrollo está basado en la cooperación y en la igualdad social (Bohman, 1996).

En relación con Chile, mientras algunos autores resaltan la tensión que ha resultado del actual énfasis acerca de lo individual -en un contexto de transformaciones económicas- en desmedro de la cultura más comunitaria en el país (Bengoa, 1996; Lechner, 2002); Araujo y Martucelli (2012) afirman que si bien el neoliberalismo ha acentuado la individuación/ privatización de las personas, esto ha ido acompañado de nuevas formas colectivas de interacción y solidaridad social. Por su parte, Goodman (2001), con su estudio respecto del posicionamiento de los chilenos a lo largo de tres bloques políticos -de derecha, centro e izquierda-, ayuda a graficar la distribución de estas orientaciones individualista -cuyos valores serían representados por la derecha- y colectiva -cuyos valores serían representados por la izquierda-. En la medida que el grupo de centro adhiere a valores individualistas y colectivos ello conforma una perspectiva más bien ecléctica. En líneas generales, de acuerdo a Goodman, la población ha tendido a agruparse en torno a estos tres bloques. En la década de los noventa, el bloque de centro aumentó significativamente agrupando en torno al cincuenta por ciento de la población. 


\subsection{MOVILIZACIONES ESTUDIANTILES COMO CONTEXTO DE CIUDADANÍA}

La reciente literatura relativa a las movilizaciones estudiantiles en Chile -iniciadas a partir del 2006- tiende a acentuar la vinculación de estas con una perspectiva colectiva de ciudadanía que integran en sus reivindicaciones una denuncia al modelo socioeconómico y político imperante (Garretón, 2007). En lo medular, estas movilizaciones se oponen al neoliberalismo económico instaurado en el país sustentado a base de relaciones comerciales individualistas y, por el contrario, abogan por una sociedad civil con capacidad de intervenir en decisiones acerca del desarrollo nacional y garantizar la igualdad social (Rojas, 2012). Por dicha razón, las movilizaciones han acuñado como propio el llamado a oponerse al lucro en el país, entendiendo por ello n sistema de desarrollo con un marcado énfasis en el mercado y en la clase empresarial, lo que ha debilitado la vida social y política del país (Mayol, 2012; Mayol y Azócar, 2012). Junto a ello, los estudiantes cuestionan profundamente a las autoridades políticas y el rol que ha cumplido el Estado, al mismo tiempo que resisten adherir a programas políticos que no garantizan su compromiso con las instituciones públicas (Nitrihual, 2009).

Las movilizaciones estudiantiles también han propuesto un ejercicio ciudadano activo que se diferencia respecto de los niveles de apatía política demostrada por los jóvenes chilenos en años anteriores (Aravena, Camelio y Moreno, 2006; Navarrete, 2008; Aceituno et al. 2009). De acuerdo con Martínez, Silva, Morandé y Canales (2010), los jóvenes han comenzado a articular su participación ciudadana en torno a su creencia en una cultura comunitaria; en su capacidad de organizarse; en su prerrogativa para disentir y expresar su opinión, mostrando a su vez menos interés en procesos electorales y de sufragio. Desde esa perspectiva, en palabras de Fernández (2013), los jóvenes han utilizado sus propios "repertorios de acción colectiva" para expresarse y utilizar el espacio público (34). A su vez, han buscado formas distintivas para organizarse por medio de asambleas y vocerías que procuran establecer liderazgos horizontales y firmemente conectados con sus bases en vistas a la discusión de demandas y la toma de decisiones (Silva y Romero, 2013).

\subsection{PROFESORES Y PERSPECTIVAS ACERCA DE LA CIUDADANÍA}

En Chile son pocos los estudios que indagan el pensamiento de los profesores respecto de temas de ciudadanía y formación ciudadana. Al respecto, resaltan los trabajos de Flanagan, Cerda, Lagos y Riquelme (2010) y Reyes, Campos, Osandón y Muñoz (2013). Aunque la naturaleza cualitativa de estos estudios no permite una mayor generalización, coinciden en que los profesores valoran las cualidades personales de los ciudadanos, las que deben incluir, entre otras, el respeto a las normas, los derechos de las personas y la institucionalidad; la tolerancia a las diferencias culturales; y el buen trato y disposición a colaborar con los demás. Junto a ello, como lo señalan estos mismos estudios, los profesores promueven distintas formas de participación ciudadana, aunque su mayor énfasis está en el sufragio y los mecanismos de la democracia representativa. Comparada esta descripción con las perspectivas de ciudadanía reseñadas anteriormente en este artículo, pareciera que estos profesores presentarían una menor tendencia a adherirse a una visión colectiva de la ciudadanía. No obstante, la identidad política de los profesores es un aspecto mayoritariamente ausente en estos dos estudios (por ejemplo, cuál es la opinión de los docentes respecto del modelo de desarrollo vigente y cómo afecta a sus estudiantes). 
Respecto de los contextos para el desarrollo de la ciudadanía, resaltan los estudios que se focalizan en la propia escuela y en el aula y de cómo contribuyen a la formación ciudadana de los estudiantes (Inzunza, 2003; Cerda, Egaña, Magendzo, Santa Cruz y Varas, 2004). Insunza señala que estos contextos escolares, a su vez, están notablemente influidos por políticas educativas que buscan promover una identidad ciudadana menos política y orientada a adaptarse a las demandas del mundo adulto centrado en el trabajo. Junto a estas políticas, los profesores tienden a no integrar los modos de expresión propios de la realidad juvenil y a mirar con sospecha la vida política de sus estudiantes, ya sea por su activismo asociado a la violencia o por su apatía asociada al individualismo. En contraste, señala Insunza, profesores con un trasfondo participativo en organizaciones sociales tienden a ser críticos con esta institucionalidad escolar, y favorecer la integración de las experiencias de los estudiantes a su formación ciudadana.

\subsection{FORMACIÓN PARA LA CIUDADANÍA EN EL CURRÍCULO NACIONAL}

Aun cuando la formación ciudadana se encuentra establecida en los Objetivos Fundamentales Transversales del currículo chileno a ser incorporados en las diferentes disciplinas de enseñanza (MINEDUC, 2009), resalta su vinculación con el sector curricular de la Historia, Geografía y Ciencias Sociales en donde el estudiante adquiere la comprensión del mundo social y político-democrático que le rodea (Magendzo, Donoso y Rodas, 1997). Esta dimensión contextual está explicitada en el informe de la Comisión de Formación Ciudadana (2004), que sugiere situar la formación ciudadana bajo el contexto más amplio de las transformaciones ocurridas en Chile a raíz del proceso de modernización, y que demanda ciudadanos colaborativos y con sentido de pertenencia al país. Este aspecto contextual también es señalado por el estudio Internacional de Educación Cívica y Formación Ciudadana ICCS-2009, que informa que los estudiantes chilenos de octavo básico no alcanzan una comprensión de la complejidad del sistema político con sus principios jurídicos y culturales (Gobierno de Chile, 2010). No obstante, estos estudiantes conceden gran importancia a la participación cívica (en la escuela y protestas legales) y a la promoción de igualdad social para los diversos grupos del país. Dicha constatación podría justificar la incorporación de temáticas como las movilizaciones estudiantiles en el currículo.

Mientras el énfasis en mediciones estandarizadas pareciera llevar a los establecimientos educativos a focalizarse en disciplinas como el lenguaje y las matemáticas en desmedro de áreas de aprendizajes como la formación ciudadana (Del Solar, 2009), no existen estudios acabados que den cuenta del nivel de integración y presencia de la formación ciudadana en el currículo, y si en aquello inciden los profesores. El trabajo de Magendzo y Toledo (2009), centrado en la educación de los derechos humanos a la luz de la historia reciente en el país, es fundacional al respecto. Entre sus conclusiones señala que entre los profesores de Historia y Ciencias Sociales de la Región Metropolitana que participaron en este estudio existe una diferencia significativa entre su afinidad política (de izquierda o de derecha) y la enseñanza los derechos humanos. En palabras de Magendzo y Toledo, "mientras más de izquierda se define el profesor, declara mayor intención de enseñar los derechos humanos" (2009: 151). Sin embargo, no se precisa qué significa ser de izquierda y cuáles son los conceptos claves que los profesores vinculan a los derechos humanos. Tampoco se menciona si los profesores integran la experiencia en derechos humanos de los propios alumnos. 


\subsection{PREDICCIONES}

En esta investigación se evaluarán cuatro predicciones que buscan establecer la relación que existe entre el pensamiento de profesores de Historia acerca de la ciudadanía con su valoración del movimiento estudiantil y la inclusión de esta temática en sus prácticas pedagógicas.

Las predicciones 1 y 2 tienen naturaleza confirmatoria. Por tanto buscan entregar sustento empírico al vínculo existente entre el pensamiento de los profesores y las perspectivas colectivas e individualistas que existen en la ciudadanía; y acerca del ejercicio cívico activo. El cumplimiento de las predicciones 1 y 2, permitirá sustentar de forma más robusta las predicciones 3 y 4, que se centran explícitamente en la valoración de las movilizaciones estudiantiles y en la decisión de los profesores de incluir o no el tema de las movilizaciones estudiantiles en el currículum. De esta manera:

1. Considerando los aportes de la literatura (Walzer, 1990; Van Gunsteren, 1994). respecto de la tensión existente entre perspectivas individualistas y colectivas de la ciudadanía, la primera predicción intentará confirmar por medio de la aplicación de cuestionarios la existencia de una asociación inversa entre la adhesión por parte de los profesores a una perspectiva individualista y la adhesión a una perspectiva colectiva de ciudadanía. De esta forma se intentará establecer que aunque ambas concepciones pueden estar presentes en una misma persona -por ejemplo en una perspectiva ecléctica-, sin lugar a dudas estas concepciones tienden a presentarse como opciones opuestas.

2. En segundo lugar, al considerar la importancia que la perspectiva colectiva concede al ejercicio cívico activo (Young, 1996; Barber, 2003), se espera encontrar una asociación positiva entre la adhesión a una perspectiva colectiva de ciudadanía por parte de los profesores y su valoración de una ciudadanía activa. En contraposición, es esperable una relación inversa o simplemente que no exista asociación entre la adhesión a una perspectiva individualista de ciudadanía por parte de los profesores y su valoración de una ciudadanía activa.

3. En tercer lugar, en relación con la valoración explícita del movimiento estudiantil como contexto para el desarrollo de una ciudanía activa, se podrá establecer una asociación de tipo positiva entre la adhesión a una perspectiva colectiva de ciudadanía por parte de los profesores y su valoración del movimiento estudiantil para el desarrollo de una ciudadanía activa. En este sentido se espera una relación inversa entre la valoración del movimiento estudiantil para el desarrollo de una ciudadanía activa y la perspectiva individualista.

4. Finalmente, se espera que los profesores que han incluido el tema de las movilizaciones estudiantiles en el currículum, en comparación con los que no lo hacen, adhieran de manera más intensa a la perspectiva colectiva de la ciudadanía, valoren la ciudadanía activa, y valoren las movilizaciones estudiantiles como contexto para el desarrollo de una ciudadanía activa. No obstante, no se espera necesariamente que los profesores que adhieran a una perspectiva individualista, que no valoren la ciudadanía activa o que presenten una menor valoración del movimiento estudiantil, no integren esta temática en el currículo. Adicionalmente, también se espera que los profesores que trabajan en establecimientos educacionales cuyos centros de alumnos participaron en las movilizaciones estudiantiles presenten una mayor tendencia a incorporar la temática en su currículo. 


\section{METODOLOGÍA}

\subsection{PARTICIPANTES}

La muestra se compuso de 152 profesores de ambos sexos (hombres: $\mathrm{N}=69$, mujeres: $\mathrm{N}=83$ ) que imparten clases en enseñanza media (es decir, secundaria), del sector de Historia, Geografía y Ciencias Sociales del currículo chileno. Los profesores participantes de este estudio respondieron a los cuestionarios de manera remota a partir de una plataforma online. Los participantes provenían de 11 ciudades que son capitales de regiones en las que se divide Chile (es decir, Arica $=7,2 \%$; Iquique $=7,9 \%$; Antofagasta $=7,2 \%$; La Serena $=7,9 \%$; Valparaíso $=15,1 \%$; Rancagua $=6,6 \%$; Talca $=9,2 \%$; Concepción $=$ 7,2\%; Temuco $=6,6 \%$; Valdivia $=6,6 \%$; Puerto Montt $=8,6 \%$; Santiago $=9,9 \%$ ). Cada uno accedió a participar de forma voluntaria en el estudio y no se entregaron compensaciones económicas por la participación.

Siguiendo la tipología general que clasifica a los establecimientos educativos chilenos, de estos 539 establecimientos el 14\% eran privados (financiados y administrados por privados); $24 \%$ eran públicos (gratuitos y administrados por el Estado); y el 62\% eran subvencionados (financiados en parte por el Estado y administrados privadamente). De los profesores que completaron los cuestionarios, $27 \%$ proviene de establecimientos educativos públicos, $29 \%$ de privados, y $44 \%$ de subvencionados. Junto a ello, $54 \%$ son de sexo femenino y $45 \%$ masculino. Finalmente, $46 \%$ de los profesores llevan entre 0 y 10 años de experiencia docente, $26 \%$ lleva entre 10 y 20 años y el restante $28 \%$ lleva más de 20 años.

\subsection{INSTRUMENTO}

La recolección de datos se realizó con un cuestionario en línea durante los meses de mayo a julio 2013. Este cuestionario fue diseñado ad hoc, validado por un grupo de expertos y sometido a una prueba piloto (Sanhueza, Cardona y Friz, 2012). El cuestionario está compuesto por cuatro instrumentos:

1. Adhesión a una perspectiva individualista: el cuestionario se construye a partir de cuatro ítems que se responden en formato de tipo Likert de 5 valores ( 1 = Completamente en desacuerdo, 5 = Completamente de acuerdo). El cuestionario consta de un factor que evalúa la adhesión de los profesores a una perspectiva individualista de ciudadanía. Para ello se considera su preferencia por un Estado que resguarde el bien privado; por un modelo de desarrollo basado en el desarrollo individual; y su pensamiento acerca de si las movilizaciones estudiantiles consideran los dos aspectos anteriores desde una perspectiva individualista (p.ej., las movilizaciones estudiantiles han ayudado a que los jóvenes le exijan al Estado resguardar el bien/interés privado). Un posterior análisis factorial exploratorio respalda esta estructura con 49,60\% de la varianza explicada para el primer factor (Eigenvalues: Factor 1: 1.98, Factor 2: ,93, Factor 3: ,69, etc.) que incluye las cuatro preguntas (Matriz de Componentes: ,69, ,70, ,71, ,70). El valor $\alpha$ de Cronbach para el cuestionario es de ,66.

2. Adhesión a una perspectiva colectiva: el cuestionario se construye a partir de tres ítems que se responden en formato de tipo Likert de 5 valores $(1=$ Completamente en desacuerdo, 5 = Completamente de acuerdo). El cuestionario consta de un factor que 
evalúa la adhesión de los profesores a una perspectiva colectiva de ciudadanía. Para ello se considera su preferencia por un modelo basado en el desarrollo en conjunto de la ciudadanía; y su pensamiento concerniente a si las movilizaciones estudiantiles favorecen dicho modelo de desarrollo y un rol del Estado centrado en el bien común. Aunque el concepto de bien común también es definido desde una perspectiva individualista (por ejemplo, el bien común es la suma agregada de intereses privados), no se pregunta por la adhesión de los profesores a un rol del Estado centrado en el bien común. No obstante, este aspecto queda subsumido en el ítem referido a si las movilizaciones estudiantiles han ayudado a que los jóvenes le demanden al Estado proteger el interés/bien común, porque los estudiantes explícitamente han definido el bien común en oposición a los intereses privados. Un posterior análisis factorial exploratorio respalda esta estructura con $60,43 \%$ de la varianza explicada para el primer factor (Eigenvalues: Factor 1: 1.81, Factor 2: ,94, Factor 3: ,24 etc.) que incluye las tres preguntas (Matriz de Componentes: 3, ,36, ,90). El valor $\alpha$ de Cronbach para el cuestionario es de ,67.

3. Importancia de la ciudadanía activa: el cuestionario consta de cinco preguntas que se responden en una escala Likert de cinco valores $(1$ = Completamente en desacuerdo, 5 = Completamente de acuerdo). El cuestionario consta de dos factores que evalúan la adhesión de los profesores a una ciudadanía activa sustentada en la deliberación y en la participación. Un factor evalúa a nivel conceptual (p.ej., los ciudadanos deberían participar en iniciativas y proyectos políticos y sociales), y el otro factor a nivel del ejercicio cívico (p.ej., participo en iniciativas o proyectos políticos y sociales). Un posterior análisis factorial exploratorio respalda esta estructura con 39,62\% de la varianza explicada para el primer factor y 30,23\% de la varianza explicada para el segundo factor que incluye tres preguntas (eigenvalues: Factor 1: 1,98, Factor 2: 1,51, Factor 3: ,61, etc.). De esta manera, el primer factor es denominado "opinión sobre ciudadanía activa" (Componentes: ,77, ,81, ,76) y el segundo factor "ejercicio activo" (componentes: ,82, ,85). El valor $\alpha$ de Cronbach para la opinión acerca de ciudadanía activa es de $\alpha=, 69$; y para el ejercicio activo de $\alpha=, 72$. Ambas escalas no están correlacionadas entre sí $(\mathrm{r} s=, 02, \mathrm{p}=, 79)$.

4. Valoración del movimiento estudiantil para la ciudadanía activa: el cuestionario evaluó la valoración que tienen los docentes del movimiento estudiantil en relación con una ciudadanía activa en función de tres virtudes cívicas: mantenerse informados, deliberación y participación en iniciativas cívicas (p.ej., las movilizaciones han ayudado a que los alumnos se involucren en iniciativas o proyectos políticos y sociales). Dicho cuestionario consta de tres preguntas que se responden en una escala Likert de cinco valores $(1=$ Completamente en desacuerdo, $5=$ Completamente de acuerdo $)$. El cuestionario consta de un factor: valoración del movimiento estudiantil. Un posterior análisis factorial exploratorio respalda esta estructura con 83,59\% de la varianza explicada para el primer factor que incluye las tres preguntas (Eigenvalues: Factor 1: 2,50, Factor 2: 31, Factor 3: ,17, etc.). De esta manera, el factor es denominado "Valoración del movimiento estudiantil" (Componentes: ,92, ,93, ,88). El valor $\alpha$ de Cronbach para el cuestionario es de, 90 .

Finalmente también se solicitó a los participantes responder de manera dicotómica (sí/no) si habían incluido o no las movilizaciones estudiantiles como un tema a tratar 
dentro de los contenidos programáticos de la asignatura de Historia y Ciencias Sociales; y si el Centro de Alumnos de sus colegios había participado o no activamente en las movilizaciones estudiantiles.

\subsection{ANÁLISIS ESTADÍSTICOS}

Se optó finalmente por utilizar estadística no paramétrica para evaluar las hipótesis, ya que las variables no presentaron una distribución normal y fueron resistentes a transformaciones. De esta manera, se aplicaron correlaciones de Spearman de dos colas para estimar las asociaciones entre variables (i.e., para las predicciones 1, 2 y 3). También se utilizaron pruebas U-Mann Whitney para comprobar diferencias entre los grupos, así como también la prueba $\mathrm{X}^{2}$ para comprobar diferencias entre variables categóricas (i.e., predicción 4).

\section{RESULTADOS}

Los valores descriptivos de las variables se resumen en la Tabla 1. Los resultados referidos a las hipótesis se presentan a continuación:

En relación con la primera predicción, se encontró una asociación inversa ( $\mathrm{r} s=-, 17$, $\mathrm{p}<, 05$ ) entre la adhesión de los profesores a una perspectiva individualista y a una colectiva (considerando el rol del Estado, el modelo de desarrollo y las movilizaciones estudiantiles). De esta manera, mientras la adhesión a una perspectiva colectiva se acrecienta, disminuye la perspectiva individualista. Más explícitamente, en la medida que en los profesores hay una mayor tendencia a adherir a una concepción colectiva, presentan una menor tendencia a adherir a una concepción individualista.

En cuanto a la evaluación de la segunda predicción, la adhesión de los profesores a una perspectiva individualista no se asoció a una preferencia por una ciudadanía activa tanto a nivel conceptual ( $\mathrm{r} s=-, 07, \mathrm{p}=, 38$ ) como en términos de su ejercicio cívico efectivo ( $\mathrm{r} s=, 08, \mathrm{p}=, 35$ ). En contraste a estos resultados, la adhesión de los profesores a una perspectiva colectiva se asoció positivamente a su preferencia por una ciudadanía activa a nivel conceptual ( $\mathrm{r} s=, 19, \mathrm{p}<, 05)$. No obstante, no se pudo establecer una relación entre la adhesión a una perspectiva colectiva con el ejercicio cívico efectivo de los profesores ( $\mathrm{r} s=, 04, \mathrm{p}=, 57$ ).

La tercera predicción relacionada con la asociación negativa que se esperaba entre la adhesión de los profesores a una perspectiva individualista con la valoración del movimiento estudiantil para la ciudadanía activa no pudo ser constatada $(r s=-, 10, p=, 23)$. De esta manera, no se puede determinar que una mayor adhesión individualista conlleve una menor valoración del movimiento estudiantil. Sin embargo, la adhesión de los profesores a una perspectiva colectiva mostró una robusta y positiva asociación con la valoración del movimiento estudiantil como contexto para el desarrollo de una ciudadanía activa ( $\mathrm{r} s=, 56, \mathrm{p}<, 001)$.

Finalmente, para la evaluación de la cuarta predicción se evaluó si las movilizaciones estudiantiles eran incluidas por los profesores en sus contenidos curriculares y su relación con los cuestionarios. De esta forma, se encontró que los profesores incluyen mayoritariamente el movimiento estudiantil en sus contenidos curriculares (Inclusión: $\mathrm{N}=122$; No inclusión: $\mathrm{N}=30$; Prueba Binomial: $\mathrm{p}<, 001$ ). Sin embargo, no se pudo 
establecer $\left(\mathrm{X}^{2}=1,751, \mathrm{p}=, 186\right)$ que aquellos profesores que trabajan en establecimientos educacionales cuyos Centros de Alumnos participaron en las movilizaciones estudiantiles $(\mathrm{N}=51)$ presentasen una mayor tendencia a incorporar la temática de las movilizaciones en el currículum que aquellos que estaban en establecimientos en los cuales los Centros de Alumnos no participaron $(\mathrm{N}=101)$.

Respecto de los cuestionarios (Fig. 1), se encontraron diferencias significativas (U $=1.378,50, \mathrm{p}<, 05)$ en la relación entre la inclusión en el currículo de la temática del movimiento estudiantil y quienes valoran las movilizaciones estudiantiles como contexto para la ciudadanía activa. Así (fig. 1), aquellos profesores que incluyen las movilizaciones estudiantiles en el currículum a su vez las valoran con mayor intensidad que quienes no lo hacen (Inclusión: $\mathrm{M} \pm \mathrm{SD}=12,31 \pm 2,83$; No inclusión: 11,16 $\pm 3,14$ ). Además, la adhesión a la perspectiva colectiva está próxima a ser significativamente mayor $(\mathrm{U}=$ $1.462,50, \mathrm{p}=, 07)$ para la inclusión de las movilizaciones estudiantiles en el currículum respecto de su no inclusión (Inclusión: 13,39 $\pm 2,05$; No inclusión: 12,50 $\pm 2,62$ ). En cuanto a la adhesión a la perspectiva individualista, esta no arrojó diferencias significativas $(\mathrm{U}=1.764,50, \mathrm{p}=, 76)$ en relación a la inclusión o no de las movilizaciones estudiantiles como temática en el currículo (Inclusión: 10,78 \pm 4,22; No inclusión: 11,06 $\pm 3,81$ ). Por lo demás, no hubo diferencias entre profesores respecto de si incluían la temática en el currículum considerando los distintos niveles de importancia que le concedían a la ciudadanía activa, ya sea a nivel conceptual $(\mathrm{U}=1.750, \mathrm{p}=, 519$; Inclusión: 9,78 \pm ,752; No inclusión: 9,83 $\pm 3,79$ ) como a nivel de su ejercicio cívico ( $\mathrm{U}=1.650,50, \mathrm{p}=$ ,403; Inclusión: 9,99 \pm 2,64; No inclusión: 10,46 $\pm 2,75$ ).

Tabla 1. Estadísticos descriptivos $(\mathrm{M} \pm \mathrm{SD})$ de las variables utilizadas en la investigación

\begin{tabular}{|l|c|}
\hline \multicolumn{1}{|c|}{ Variables } & M \pm DE* \\
\hline Perspectiva individualista del rol del Estado y de las movilizaciones estudiantiles & $10,84 \pm 4,14$ \\
\hline Perspectiva colectiva del rol del Estado y de las movilizaciones estudiantiles & $13,21 \pm 2,19$ \\
\hline Importancia de la ciudadanía activa: concepciones docentes & $9,79 \pm .69$ \\
\hline Importancia de la ciudadanía activa en el ejercicio cívico de los profesores & $10,08 \pm 2,66$ \\
\hline Valoración del movimiento estudiantil & $12,08 \pm 2,92$ \\
\hline
\end{tabular}

Nota: $\mathrm{N}=152 . \mathrm{M} \pm \mathrm{DE}=$ Media y deviación estándar.

\section{DISCUSIÓN}

En esta investigación se indagó en la relación entre el pensamiento de profesores de Historia acerca de la ciudadanía y la inclusión de la temática de las movilizaciones estudiantiles en su enseñanza. Para establecer esta relación el estudio planteó cuatro predicciones, siendo las dos primeras de naturaleza confirmatoria, y las últimas dos de corte exploratorio para analizar cuantitativamente esta problemática en Chile. Los resultados obtenidos permiten dar sustento parcial a las predicciones planteadas. En este sentido, es importante resaltar que la perspectiva colectiva se comportó de manera más robusta para las diferentes predicciones. De esta manera, esta perspectiva se asoció de la manera 
Figura 1. Diferencias en la inclusión en el currículum del movimiento estudiantil en relación con los cuestionarios aplicados

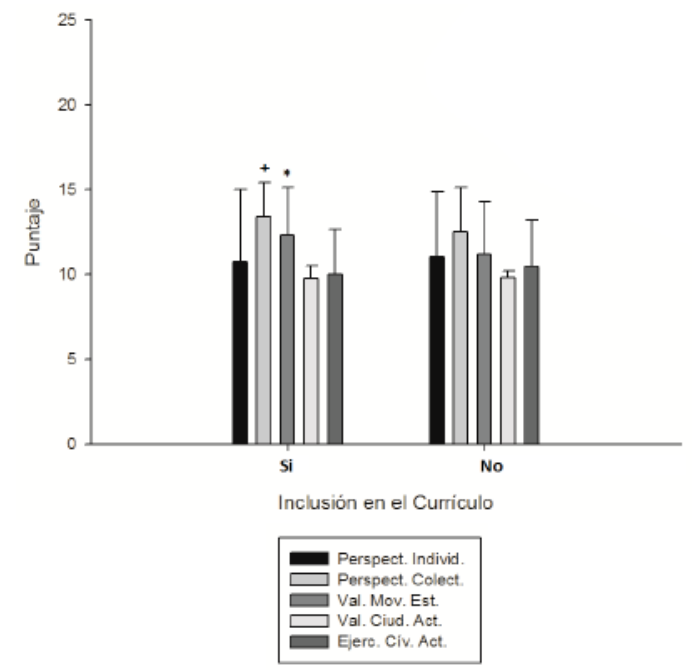

Nota: $* \mathrm{p}<, 05 ;+\mathrm{p}<, 08$. Perspect. Individ. = Perspectiva Individualista; Perspect. Colect. = Perspectiva Colectiva; Val. Mov. Est. = Valoración del Movimiento Estudiantil; Val. Ciud. Act. Valoración Ciudadanía Activa; Ejerc. Civ. Act. Ejercicio Cívico Activo.

esperada por las predicciones a la mayoría de las variables estudiadas, aunque no mostró diferencias en relación con la inclusión de las movilizaciones estudiantiles en el currículo. En consecuencia, la perspectiva individualista no se pudo asociar a la gran mayoría de las variables estudiadas, ni tampoco presentó diferencias entre grupos al realizar comparaciones en relación con la inclusión del movimiento estudiantil en el currículo. Estos resultados deben ser necesariamente analizados considerando detalladamente cada una de las predicciones y el valor que en su conjunto poseen todos los resultados.

De acuerdo con los resultados se confirma la primera hipótesis del estudio respecto de que las perspectivas de ciudadanía (colectivas e individualistas) presentadas en la literatura e integradas en las opiniones de los profesores tienden a asociarse negativamente. Este tipo de asociación permitiría confirmar, a su vez, que los profesores distinguen ambas perspectivas, y que tal como se ha discutido en este trabajo tienden a considerarlas en tensión. En la medida que los estudios concernientes a las concepciones de ciudadanía de los profesores tienden a considerar su pensamiento en relación con las virtudes y actitudes que hacen al buen ciudadano (Davies et al., 1999; Flanagan et al., 2010), los resultados de este estudio son una contribución, ya que establecen la relación entre el pensamiento de los profesores y conceptos provenientes de la teoría política, como el modelo de desarrollo, el rol del Estado y los bienes e intereses comunes y privados. Los resultados asimismo sugieren que aunque los profesores de este estudio presentan una tendencia mayoritaria a adherir a una perspectiva colectiva, no presentan un rechazo absoluto a una perspectiva individualista. Lo anterior podría sugerir que los profesores tienden a perspectivas más eclécticas, aunque valoricen algunos polos más que otros (Goodman, 2001; Araujo y Martucelli, 2012). 
La segunda predicción es validada en parte por los resultados del estudio. Por un lado no hay una asociación entre adhesión a una perspectiva individualista y una valoración de la ciudadanía activa. Por otro lado se observa una asociación positiva entre la adhesión a una perspectiva colectiva y la valoración del ejercicio cívico activo a nivel conceptual. No obstante, no existe asociación entre dicha adhesión conceptual y el ejercicio cívico activo desarrollado por los profesores. Las razones varían en la literatura siendo la intensificación de la actividad docente -que no deja tiempo para que los profesores realicen otro tipo de actividades- un factor mencionado recurrentemente (Apple, 2004). De esta manera, aunque los profesores quisiesen ser más participativos a nivel social (p. ej., en de juntas de vecinos, clubes deportivos, etc.), esta participación está en entredicho producto de la alta carga laboral. De hecho, según un reciente censo efectuado a los profesores chilenos (Eduglobal, 2012), el 31\% de ellos señaló tener menos de tres horas semanales para dedicar a actividades de esparcimiento. Estudios posteriores debieran verificar esta incongruencia entre el pensamiento y el actuar de los profesores en materia de ciudadanía, tomando en cuenta un mayor número de factores asociados a la profesión.

La tercera predicción es igualmente validada en parte por el estudio. Por un lado se comprobó una robusta asociación entre la perspectiva colectiva y la valoración del movimiento estudiantil como contexto para el desarrollo de la ciudadanía activa. Estos resultados se condicen con la literatura reseñada en este artículo, en la medida que perspectivas teóricas que subrayan la necesidad de lo colectivo enfatizan la importancia de la participación ciudadana (por ejemplo, Barber, 2003), un aspecto que las movilizaciones estudiantiles han igualmente subrayado. No obstante, contrario a la predicción inicial, no existe una asociación negativa entre una adhesión a una perspectiva individualista y la valoración del movimiento estudiantil. En ese sentido, aunque tampoco hay una asociación positiva, la adhesión a una perspectiva individualista no implica desvalorizar el movimiento estudiantil. En parte, esto se podría explicar en la medida que una ciudadanía activa no tan solo se valoriza bajo determinados supuestos políticos, sino también, por ejemplo, como instancia de crecimiento en virtudes personales -empatía, generosidad, compromiso, etc.-, lo que de hecho podría estar en consonancia con presupuestos de corte individualista (Westheimer \& Kahne, 2004).

Los resultados anteriores sustentan la verificación de la cuarta predicción, sustancial al objetivo de este estudio, y que consiste en indagar la relación que existe entre el pensamiento de los profesores de Historia acerca de la ciudadanía y la inclusión de esta temática en sus prácticas pedagógicas. De este modo, la consideración de las distintas variables permite establecer de mejor manera la especificidad de esta relación. De acuerdo con los datos obtenidos, porque la mayoría de los profesores tienden a incorporar la temática de las movilizaciones estudiantiles en su currículo, pareciera que las diferencias en sus concepciones de ciudadanía no tienen una influencia decisiva (i.e., profesores tanto con una tendencia hacia una perspectiva individualista como colectiva incorporan la temática, aunque existe una ligera mayor probabilidad de que lo hagan aquellos que adhieren a una perspectiva colectiva). Asimismo, aunque la mayoría de los profesores tienden a adherir a una ciudadanía activa o a presentar en su mayoría niveles menores de ejercicio cívico activo, no es posible establecer si estas variables inciden decisivamente en la incorporación de la temática en el currículo (i.e. profesores que incluyen la temática en el currículo como aquellos que no adhieren a una ciudadanía activa). 
Desde esa perspectiva, el factor asociado a la inclusión de la temática de las movilizaciones estudiantiles en el currículo que más resalta en el pensamiento de los profesores se refiere a la valoración de estas movilizaciones como contexto de desarrollo cívico. El hecho de que los Centros de Alumnos de sus establecimientos educativos hayan participado activamente o no en las movilizaciones, no se asocia decisivamente en su decisión de enseñar la temática. Por tanto se puede colegir que una experiencia más cercana a las movilizaciones estudiantiles no necesariamente lleva a los profesores a incorporar el tema a su enseñanza de la ciudadanía. En este sentido, el pensamiento de los profesores adquiere gran relevancia en relación con la incorporación de la temática en el currículo en la medida que contenga una valorización de las movilizaciones estudiantiles (que no necesariamente se explique por una experiencia directa con ellas), y que esta valorización tienda a sustentarse en una comprensión teórica de la ciudadanía desde una perspectiva colectiva; es decir, desde un pensamiento que subraye el desarrollo en conjunto de la sociedad.

\section{COMENTARIOS FINALES}

Los resultados presentados revelan que las movilizaciones estudiantiles son efectivamente una temática que los profesores en su mayoría incluyen en su enseñanza y que, por tanto, podría esperarse que la consideren adecuada como parte de la formación ciudadana que debe entregarse a los alumnos. Junto a ello se establece la relación positiva que aquello tiene con el pensamiento de los propios profesores, especialmente referido a una valorización de estas movilizaciones como contexto de desarrollo cívico y si esta valorización está asociada a la adhesión de una perspectiva colectiva acerca de ciudadanía. En ese sentido, el presente estudio centra su análisis desde las concepciones de los profesores, en vez de focalizarse en los contenidos curriculares establecidos en los libros de texto en uso o sugeridos por los programas oficiales del sistema escolar (Gimeno, 1998).

El énfasis en la relevancia del pensamiento de los profesores del presente estudio resalta la existencia de un recurso pedagógico que facilita la incorporación al aula de una temática afín a los estudiantes, en momentos en que el énfasis en exámenes estándares disminuye los espacios curriculares para áreas de formación, como la educación para la ciudadanía (Kahne \& Westheimer, 2003). Este recurso pedagógico no tan solo se refiere a que los docentes muestren la disponibilidad para incorporar el tema en el currículo, sino a disponer de perspectivas teóricas que, al estar en sintonía general con los estudiantes, ayuda a generar un ambiente de aprendizaje propicio para la discusión de temáticas políticas, aterrizar los contenidos de enseñanza a la realidad y experiencias de los estudiantes, así como favorecer un diálogo pedagógico que permita profundizar conceptos que son fundamentales para toda la ciudadanía como el rol del Estado, el desarrollo del país y el bien común (Hess, 2002).

El enfoque temático de la presente investigación es igualmente una contribución a los estudios realizados en Chile que, como han sido reseñados en el presente artículo, presentan algunas limitaciones en su alcance temático. Aunque el presente estudio incorpora conceptos relativos al comportamiento cívico y su conexión con prácticas pedagógicas habitualmente considerados en la literatura, también se incorporan nociones de teoría política a partir de dos temas de máxima contingencia en Chile: el rol del 
Estado y el modelo de desarrollo nacional. Sin embargo se reconocen al mismo tiempo las limitaciones planteadas por Goodman (2001) cuando se utiliza un diseño cuantitativo para analizar temas tan abstractos como lo son las concepciones de teoría política de los profesores. Por consiguiente, se reconoce la necesidad de complementar este estudio en el futuro con una aproximación cualitativa que permita clarificar y profundizar los resultados obtenidos hasta el momento.

Debido a que el estudio sugiere que existe disponibilidad por parte de los profesores para incorporar la temática en el aula, así como que esto está asociado con una valoración positiva de las experiencias que hoy los estudiantes viven en el ámbito cívico, es importante que el sistema en su conjunto también reconozca y fortalezca la integración de estos contenidos curriculares en la formación ciudadana de los estudiantes, dando los apoyos teóricos y pedagógicos correspondientes. Este llamado resalta en momentos en que se cuestiona la falta de tratamiento en las aulas chilenas de temáticas contingentes pertenecientes a la realidad del país (Smink, 2013). Hacer este llamado en un contexto nacional que exige replantear las nociones de lo público y lo privado en el país (Atria, Larraín, Benavente, Couso y Joignant, 2013) y que ha favorecido que los estudiantes adquieran un mayor sentido cívico, constituye una contribución innegable para continuar formando jóvenes que precisamente, por medio de su ejercicio cívico, procuren seguir indagando pertinente a la relevancia del bien común en la vida del país.

\section{REFERENCIAS}

Aceituno, R., Asún, R., Ruiz, S., Reinoso, A., Venegas, J. I. y Corbalán, F. (2009). Anomia y alienación en estudiantes secundarios de Santiago de Chile: Resultados iniciales de un estudio comparativo 1989-2007. Psykhe, vol. 18 (2), 3-18.

Aravena, A., Camelio, F. y Moreno, A. (2006). Generación Mayo de 2006: ¿Reivindicando el ejercicio de la ciudadanía? Revista del Observatorio de Juventud, vol. 3 (11), 6-15.

Araujo, K. y Martucelli, D. (2012). Desafios comunes. Retrato de la sociedad chilena y sus individuos (Tomo 1). Santiago: LOM.

Apple, M. (2004). Creating difference: Neo-liberalism, neo-conservatism and the politics of educational reform. Educational Policy, vol. 18 (1), 12-44.

Atria, F., Larraín, G., Benavente, J. M., Couso, J. y Joignant, A. (2013). El otro modelo. Del orden neoliberal al régimen de lo público. Santiago: Debate.

Barber, B. (2003). Strong democracy. Participatory politics for a new age. Berkeley: University of California Press.

Becker, G. (1976). The economic approach to human behavior. Chicago: The University of Chicago Press.

Beiner, R. (1995). Why citizenship constitutes a theoretical problem in the last decade of the twentieth century. In R. Beiner (Ed.), Theorizing citizenship (pp. 1-28). New York: State University of New York Press.

Bengoa, J. (1996). La comunidad perdida. Santiago: Ediciones Sur.

Bishop, J. J. \& Hamot, G. (2001). Democracy as a cross-cultural concept: Promises and problems. Theory and Research in Social Education, vol. 29 (3), 463-487.

Bobbio, N. (1987). The future of democracy. A defense of the rules of the game. Cambridge: Polity Press.

Bohman, J. (1996). Public deliberation. Pluralism, complexity, and democracy. Cambridge: MIT Press. 
Cabalin, C. (2012). Neoliberal education and student movements in Chile: Inequalities and malaise. Policy Futures in Education, vol. 10 (2), 219-228.

Cerda, A. M., Egaña, M. L., Magendzo, A., Santa Cruz, E. y Varas, R. (2004). El complejo camino de la formación ciudadana: Una mirada a las prácticas profesores. Santiago: LOM.

Chin, K. \& Barber, C. (2010). A multidimensional exploration of teachers' beliefs about civic education in Australia, England, and the United States. Theory and Research in Social Education, vol. 38 (3), 385-427.

Comisión Formación Ciudadana (2004). Informe comisión formación ciudadana. Santiago: MINEDUC.

Davies, I., Gregory, I. \& Riley, S. (1999). Good citizenship and educational provision. London: Falmer Press.

Del Solar, S. (2009). Los docentes frente al SIMCE: Entre la resistencia y la adaptación. Estudio de caso en una escuela municipal de una comuna popular santiaguina. Docencia, (38), 44-56.

Eduglobal (2012). Primer censo docente de Chile. Recuperado el 14 de enero de 2014 desde http://www.eduglobal.cl/content_edu/documents/informe_preliminar.pdf

Fernández, R. (2013). El espacio público en disputa: Manifestaciones políticas, ciudad y ciudadanía en el Chile actual. Psicoperspectivas. Individuo y Sociedad, vol. 12 (2), 28-37.

Flanagan, A., Cerda, G., Lagos, D. y Riquelme, S. (2010). Tensiones y distensiones en torno a la ciudadanía y formación ciudadana: Comparación de los significados de profesores y estudiantes secundarios en la región de Valparaíso. Última Década, (33), 115-137.

Garretón, M. (2007). Del postpinochetismo a la sociedad democrática. Globalización y política en el bicentenario. Santiago: Debate.

Gimeno, J. (1998). El currículum: Una reflexión sobre la práctica. Madrid: Morata.

Gobierno de Chile (2010). Estudio internacional de educación cívica y formación ciudadana (ICCS 2009). Primer informe nacional de resultados. Santiago: Gobierno de Chile.

Goodman, L. (2001). Chilean citizens and Chilean democracy: The management of fear, division and alienation. In R. A. Camp (Ed.), Citizen views of democracy in Latin America (pp. 206-221). Pittsburgh: University of Pittsburgh Press.

Gould, C. (1990). Rethinking democracy. Freedom and social cooperation in politics, economy, and society. Cambridge: Cambridge University Press.

Habermas, J. (1996). Three normative models of democracy. In S. Benhabib (Ed.), Democracy and difference: Contesting the boundaries of the political (pp. 21-30). New Jersey: Princeton University Press.

Hayek, F. (1960). The constitution of liberty. Chicago: University of Chicago Press.

Held, D. (2006). Models of democracy. California: Stanford University Press.

Hess, D. (2002). Discussing controversial public issues in secondary social studies classrooms: Learning from skilled teachers. Theory and Research in Social Education, vol. 30 (1), 10-41.

Inzunza, J. (2003). Los centros de alumnos de enseñanza media: La ciudadanía del mundo feliz. Última Década, (18), 153-173.

Kahne, J. \& Westheimer, J. (2003). Teaching democracy: What school need to do. Phi Delta Kappan, vol. 85 (1), 34-40; 57-66.

Lechner, N. (2002). Las sombras del mañana. La dimensión subjetiva de la política. Santiago: LOM.

Macpherson, C. (1977). The life and times of liberal democracy. Oxford: Oxford University Press.

Magendzo, A. y Toledo, M. (2009). Educación en derechos humanos: Currículum historia y ciencias sociales del segundo año de enseñanza media, subunidad régimen militar y transición a la democracia. Estudios Pedagógicos, vol. 35 (1), 139-154.

Donoso, P. y Rodas, M. T. (1997). Los objetivos transversales en educación. Santiago: Editorial Universitaria.

Martínez, M. L., Silva, C., Morandé, M. y Canales, L. (2010). Los jóvenes ciudadanos: Reflexiones para una política de formación ciudadana juvenil. Última Década, (32), 105-118. 
Mayol, A. (2012). No al lucro. De la crisis a la nueva era política. Santiago: Debate. - y Azócar, C. (2012). Politización del malestar, movilización social y transformación ideológica: El caso Chile 2011. Polis, (30), 2-19.

MINEDUC (2009). Objetivos fundamentales y contenidos mínimos obligatorios de la educación básica y media. Santiago: Ministerio de Educación.

Mouffe, C. (2005). The democratic paradox. London: Verso.

Navarrete, B. (2008). Juventud y políticas en liceos municipales. El caso de Maipú. Última Década, (28), 167-202.

Nitrihual, L. (2009). Lo reversivo en la revolución de los secundarios en Chile. Quórum Académico, vol. 6 (1), 121-135.

Ochoa, A. (2011). Los conocimientos y opiniones de los profesores de educación primaria acerca de la asignatura de formación cívica y ética. El caso de Querétaro, México. Perfiles Educativos, vol. XXXIII (134), 115-135.

OPECH (2010). De actores secundarios a estudiantes protagonistas. Santiago: Quimantú.

Pace, J. (2008). Teaching for citizenship in $12^{\text {th }}$ grade government classes. In J. Bixby \& J. Pace (Eds.), Educating democratic citizens in troubled times: Qualitative studies of current efforts (pp. 25-57). Albany: Suny Press.

Reyes, L., Campos, J., Osandón, L. y Muñoz, C. (2013). El profesorado y su rol en la formación de los nuevos ciudadanos: Desfases entre las comprensiones, las actuaciones y las expectativas. Estudios Pedagógicos, vol. 39 (1), 217-237.

Rojas, J. (2012). Sociedad bloqueada. Santiago: RIL.

Sanhueza, S., Cardona, C. y Friz, M. (2012). La sensibilidad intercultural en el alumnado de educación primaria y secundaria de la provincia de Alicante. Perfiles Educativos, vol. XXXIV (136), 8-22.

Schumpeter, J. (1976). Capitalism, socialism, and democracy. London: Allen and Unwin.

Silva, C. y Romero, J. (2013). ¿Qué es un movimiento social fuerte? Conceptualizaciones de la noción de fortaleza y aplicación al caso del movimiento estudiantil 2002-2011 en Chile. Última Década, (38), 91-108.

Shultz, W., Ainley, J., Frailon, J., Kerr, D. \& Losito, B. (2010). ICCS 2009 International Report: Civic knowledge, attitudes, and engagement among lower-secondary school students in 38 countries. Netherlands: International Association for the Evaluation of Educational Achievement.

Smink, V. (2013). Cómo se enseña el golpe de Pinochet en las escuelas de Chile. Retrieved December 4, 2013, from http://www.bbc.co.uk/mundo/noticias/2013/09/130909_chile_golpe_escuelas_vs.shtml

Torney-Purta, J. \& Richardson, W. (2002). An assessment of what 14-year-olds know and believe about democracy in 28 countries. In W. Parker (Ed.), Education for democracy. Contexts, curricula, assessments (pp. 185-210). Greenwich, Conn: Information Age Publishing.

Touraine, A. (1997). What is democracy? Boulder: Westview Press.

Van Gunsteren, H. (1994). Four conditions of citizenship. In B. van Steenbergen (Ed.), The condition of citizenship (pp. 36-48). London: Sage.

Van Steenbergen, B. (1994). The condition of citizenship: An introduction. In B. van Steenbergen (Ed.), The condition of citizenship (pp. 1-9). London: Sage.

Urra, J. (2012). La movilización estudiantil chilena en 2011: Una cronología. OSAL, vol. XIII (31), 23-38.

Walzer, M. (1990). The communitarian critique of liberalism. Political Theory, vol. 18 (1), 6-23.

Westheimer, J. \& Kahne, J. (2004). What kind of citizen? The politics of educating for democracy. American Educational Research Journal, vol. 41 (2), 237-269.

Young, I. (1996). Communication and the other: Beyond deliberative democracy. In S. Benhabib (Ed.), Democracy and difference: Contesting the boundaries of the political (pp. 120-135). New Jersey: Princeton University Press. 